\title{
Variabilidade fenotípica e divergência genética em clones de batata doce no estado do Tocantins ${ }^{1}$
}

\author{
Phenotypic variability and genetic diversity in sweet potato clones in the state of \\ Tocantins
}

\author{
Elainy Cristina Alves Martins ${ }^{2}$, Joenes Mucci Peluzio ${ }^{3}$, Ronaldo Rodrigues Coimbra ${ }^{4}$ e Waldesse Piragé de \\ Oliveira Junior ${ }^{5 *}$
}

\begin{abstract}
RESUMO - Com objetivo de avaliar a variabilidade fenotípica e a divergência genética entre 50 clones de batata doce, foi realizado um ensaio na Estação Experimental da Universidade Federal do Tocantins - Campus de Palmas, no ano agrícola 2009/2010. A divergência genética foi avaliada pelo método de agrupamento de otimização de Tocher. As características estudadas foram produtividade, produção de matéria seca, teor de amido e rendimento de etanol. O método de otimização por Tocher separou os clones em oito grupos geneticamente distintos. As características produtividade (70,15\%) e rendimento em etanol $(15,06 \%)$ foram as que mais contribuíram na divergência genética entre os clones. A presença de variabilidade genética permitiu a identificação de cultivares dissimilares e com média elevada indicando a possibilidade de seleção de genótipos superiores para as características estudadas. Os cruzamentos Duda x 22.19, Marcela x 22.19, Barbara x 22.19, Duda x Barbara, Amanda x 22.19, Amanda x Marcela são promissores para obtenção de híbridos divergentes.
\end{abstract}

Palavras-chave: Ipomoea batatas. Biomassa. Diversidade.

\begin{abstract}
In order to evaluate the phenotypic variability and genetic divergence between 50 clones of the sweet potato, a test was conducted at the Experimental Station of the Federal Universidade of Tocantins, Palmas Campus, in the agricultural year of 2009/2010. The genetic diversity was assessed using the Tocher grouping optimization method. The characteristics studied were productivity, dry matter production, starch content and ethanol yield. The Tocher optimization method separated the clones into eight genetically distinct groups. The characteristics of productivity (70.15\%) and ethanol yield (15.06\%) were those that most affected genetic divergence among the clones. The presence of genetic variability permitted the identification of dissimilar cultivars with a high average, indicating the possibility of selecting superior genotypes for the traits studied. The Duda x 22.19, Marcela x 22.19, Barbara x 22:19, Duda x Barbara, Amanda x 22:19, Amanda x Marcela crossbreeds are those which are promising for obtaining divergent hybrids.
\end{abstract}

Key words: Ipomoea batatas. Biomass. Diversity.

\footnotetext{
*Autor para correspondência

${ }^{1}$ Recebido para publicação em 01/09/2011; aprovado em 07/05/2012

Parte da Dissertação de Mestrado do primeiro autor, apresentada ao Programa de Pós-Graduação em Agroenergia/UFT

${ }^{2}$ Laboratório de Biotecnologia/Centro Agrotecnológico/Universidade Federal do Tocantins, Campus Universitário, Palmas-TO, Brasil, biocris@ mail.uft.edu.br

${ }^{3}$ Departamento de Fitotecnia/Universidade Federal do Tocantins, Campus Universitário, Palmas-TO, Brasil, 77.001-090, joenesp@mail.uft.edu.br ${ }^{4}$ Universidade Federal do Tocantins, Campus Universitário, Porto Nacional-TO, Brasil, 77.500-000, ronaldo.rc@mail.uft.edu.br

${ }_{5}^{5}$ Laboratório de Biotecnologia/ Centro Agrotecnológico/Universidade Federal do Tocantins, Campus Universitário, Bloco de Agroenergia, Quadra 109

Norte, Av. NS 15, ALCNO 14 s/n, , Palmas-TO, Brasil, 77.001-090, waldessejunior@ mail.uft.edu.br
} 


\section{INTRODUÇÃO}

Assim como na época da crise do petróleo dos anos 70, o mundo está empenhado em encontrar uma solução duradoura para seu problema energético. A preocupação ambiental somou-se à redução dos estoques e à alta dos preços dos combustíveis fósseis para valorizar as fontes de energia renováveis e menos poluentes (PAVLAK et al., 2007). As pesquisas têm se concentrado no desenvolvimento de novos insumos básicos, de caráter renovável, para a produção de combustíveis que possam substituir os derivados de petróleo, o que coloca a biomassa em um papel de destaque, em razão da sua natureza renovável, ampla disponibilidade, biodegradabilidade e baixo custo (SUAREZ, 2007).

O Brasil apresenta características adequadas à produção de biomassa para fins energéticos, além de se destacar mundialmente como precursor desta tecnologia. Segundo Leal et al. (2010), apesar do seu potencial como matéria-prima alternativa para a produção de energia, existem poucos trabalhos de pesquisa com a cultura da batata doce, sendo que a maioria dos programas de melhoramento e seleção de genótipos visam, geralmente, características de aceitação comercial como aspecto visual. Segundo os mesmo autores, com o conceito de cultura alternativa para produção de etanol, o melhoramento e a seleção de clones de batata-doce devem buscar genótipos de alta produtividade de raízes e com alto teor de amido sem levar em consideração apenas os aspectos visuais.

A ampliação da participação da biomassa, a partir do desenvolvimento de fontes amiláceas, propicia a oportunidade de executar políticas de cunho social, ambiental e econômico em todo o território nacional. Será também uma iniciativa para promover um importante aumento de novos investimentos, emprego, renda e desenvolvimento tecnológico, além de uma oportunidade para atender parte da crescente demanda mundial por combustível de reduzido impacto ambiental (SALLA; CABELLO, 2010).

No Estado de Tocantins, a Universidade Federal de Tocantins vem desenvolvendo um programa de melhoramento de batata doce, iniciado em 1997, voltado especialmente para energia. Neste programa, foram selecionados clones de alta produtividade e alto teor de amido nas raízes (LEAL et al., 2010).

Como esta cultura pode ter fins múltiplos, torna-se necessário desenvolver e selecionar cultivares específicos para cada finalidade e incorporar nestes as principais características agronômicas desejáveis.

O sucesso de um programa de melhoramento reside na existência de variabilidade genética na produção de trabalho. Melhoristas têm recomendado, para a formação de produção-base, o intercruzamento entre cultivares superiores e divergentes. As informações múltiplas de cada cultivar é expresso em medidas de dissimilaridade, que representam a diversidade que há no conjunto de acessos cultivados (CRUZ, 2007).

Objetivou-se no presente trabalho: 1) quantificar a variabilidade genética dos clones; 2) promover o agrupamento dos clones em função da dissimilaridade genética; 3) indicar a contribuição relativa dos caracteres avaliados para a dissimilaridade genética e; 4) identificar as combinações mais promissoras para produzir recombinações superiores.

\section{MATERIAL E MÉTODOS}

O Experimento foi realizado na Estação Experimental do Campus Universitário de Palmas da Universidade Federal do Tocantins (UFT) (Latitude: 10¹0'40" S; Longitude: 48 21'43" O; Altitude: $216 \mathrm{~m}$ ). O plantio foi realizado no mês de outubro/2009 e a colheita realizada em abril/2010. Segundo o Laboratório de Meteorologia e Climatologia da UFT, a variação mensal da temperatura durante o período foi de 26 a $28^{\circ} \mathrm{C}$, a umidade relativa foi de 76,2 a $82,5 \%$ e a precipitação oscilou de 123,7 a 436,6 mm.

O delineamento experimental utilizado foi o de blocos casualizados, com 50 tratamentos e três repetições. Os tratamentos foram representados por 50 clones oriundos do Programa de Melhoramento Genético da Universidade Federal do Tocantins, sendo os seguintes: 100.06; 114.15; PA.06; Amanda; PA.18; 02.58; 114.08; 100.32; Marcela; 106.41 ; 22.32; 106.10; PA.57; Duda; 02.39; 115.02; 02.21; 106.55; CarolinaVitoria; 115.01; 114.03; 02.37; 100-18; Julia; 106.63; PA.35; 106.27; 22.06; Isabela; 04.06; 100.23; 02.26; 106.25; Beatriz; 04.12; 114.07; 48.14; PA.32; Livia; 106.79; 22.14; 112.13; 22.24; Ana Clara; 106.32; 114.22; PA.36; 22.19; Barbara; 04.30).

A parcela experimental foi composta por 10 plantas, espaçadas em 1,0 m e com uma área útil de 4,0 $\mathrm{m}^{2}$. Foram realizadas as operações de aração, gradagem e sulcamento. A adubação de plantio foi realizada conforme as exigências da cultura, após análise prévia do solo, na dosagem de $105 \mathrm{~kg} \mathrm{ha}^{-1}$ de fósforo, $90 \mathrm{~kg} \mathrm{ha}^{-1}$ de potássio e $40 \mathrm{~kg} \mathrm{ha}^{-1}$ de nitrogênio, com micronutrientes $\mathrm{B}, \mathrm{Cu}$, $\mathrm{Fe}, \mathrm{Mn}$ e $\mathrm{Zn}$.

Foram estudadas as seguintes características: a) Produção de matéria seca das plantas, obtida através da análise da umidade (ASSOCIATION OF OFICIAL ANALYTICAL CHEMISTS, 1994) e medida em porcentagem de matéria seca; b) Produtividade ( $\mathrm{t} \mathrm{ha-}{ }^{1}$ ), obtida em 10 plantas da área útil da parcela; c) Teor de amido (\%), medido através de espectrofotômetro de infravermelho próximo com resultado em porcentagem de base seca; d) 
rendimento em etanol $\left(\mathrm{m}^{3} \mathrm{ha}^{-1}\right)$, estimado a partir do teor de amido, produtividade e matéria seca dos clones.

Os dados foram submetidos à análise de variância para averiguação da variabilidade genética. Em seguida, as médias foram comparadas pelo teste de Scott e Knott (1974), com significância $\mathrm{p}<0,05$.

As medidas de dissimilaridade foram determinadas segundo o modelo de análise multivariada, o que permitiu a obtenção da matriz de dissimilaridade, da matriz de covariância residual e das médias dos cultivares. Posteriormente, foi aplicado o método de agrupamento de Tocher (RAO, 1952), utilizando a distância generalizada de Mahalanobis (D2), como medida de dissimilaridade. Utilizou-se, também, o critério de Singh (1981) para quantificar a contribuição relativa dessas características para a divergência genética.

As análises foram realizadas utilizando o programa Computacional Genes, versão 2007 (CRUZ, 2007).

\section{RESULTADOS E DISCUSSÃO}

A análise de variância para produtividade, matéria seca, teor de amido e rendimento em etanol encontra-se na Tabela 1. Pode-se verificar que ocorreram diferenças significativas entre os clones para todas as características, indicando a existência de variabilidade genética. Esses resultados evidenciam uma situação bastante favorável ao melhoramento, sugerindo a possibilidade de discriminar clones superiores. Os coeficientes de variação $(\mathrm{CV})$ foram médios para as variáveis Produtividade e Rendimento em Etanol, o que é esperado para atributos de natureza quantitativo sendo bastante influenciados pelo ambiente e foram baixos para os atributos Matéria Seca e Teor de amido, mostrando existir uma boa precisão experimental na avaliação dessas características.

Com relação à produtividade dos clones, foram formados sete grupos de médias, segundo teste de Scott e Knott (Tabela 2). O grupo com as maiores médias foi representado por dois clones, Duda (40,9 t ha- $\left.{ }^{1}\right)$ e 22.19
(45,4 $\left.\mathrm{t} \mathrm{ha}^{-1}\right)$. O segundo grupo foi representado por cinco clones, com médias entre 33,5 e $39,0 \mathrm{t} \mathrm{ha-}^{-1}$. O terceiro grupo por sete clones, apresentando valores de 28,3 a $32,3 \mathrm{t} \mathrm{ha}^{-1}$. O quarto, quinto e sexto grupo foram formados por 11,10 e 11 clones, respectivamente. $\mathrm{O}$ sétimo grupo, constituído de quatro clones, foi o que apresentou as menores médias, 6,1 a 4,4 t ha- ${ }^{-1}$. Andrade Júnior et al. (2009) selecionando clones de batata doce para a região do Alto Vale do Jequitinhonha obtiveram uma produção total de raízes que variou de 22,0 a 45,4 t ha-1 .

Cardoso et al. (2005) avaliando o desempenho de clones de batata doce em Vitória da Conquista encontraram produtividades de 4,1 a 28,5 t ha- ${ }^{-1}$ Santos et al. (2006), trabalhando com a cultivar Rainha Branca obtiveram produção máxima de $18,5 \mathrm{tha}^{-1}$.

Os valores obtidos para percentual de matéria seca das raízes (Tabela 2) variaram entre 24,53\% (clone 106.25) a $39,13 \%$ (clone PA.18), sendo formados quatro grupos. $\mathrm{O}$ primeiro grupo, representado por mais da metade dos clones, apresentou valores médios variando de 33,74 a 39,13\%, com destaque para os clones PA.18, 22.19, 106.63 e Carolina Vitoria, que apresentaram os maiores valores médios. $\mathrm{O}$ segundo grupo apresentou 18 clones, com médias entre 30,56 a $33,44 \%$. O terceiro grupo foi constituído pelos clones 48.14 e 114.15 com medias de 28,86 e $28,40 \%$ respectivamente. $\mathrm{O}$ quarto grupo, com apenas o clone 106.25 obteve a média mais baixa de matéria seca, $24,53 \%$.

Leonel e Cereda (2002) estudando a caracterização físico-química de sete tipos de tuberosas amiláceas encontraram $32,2 \%$ de matéria seca na batata doce, destacando-a das demais tuberosas avaliadas. Roesler et al. (2008) avaliando quatro cultivares de batata doce com aptidão ao processamento de fécula, encontraram valores de matéria seca que variaram de 23,63 a 33,82\%. Assim, de acordo com os autores, a cultivar CNPH 003 mostrou-se a mais indicada, em função de apresentar, dentre outras características, o maior teor de massa seca, uma vez que o maior teor desta variável proporciona uma maior extração de amido.

Tabela 1 - Análise de variância para quatro características avaliadas em 50 clones de batata doce. Palmas-TO, 2010

\begin{tabular}{lccccc}
\hline & F.V. & G.L. & \multicolumn{5}{c}{ QM } \\
\cline { 3 - 6 } & & Pr & MS & TA & RE \\
\hline Blocos & 2 & 0,4866 & 43,1539 & 3,2796 & 0,0242 \\
Tratamentos & 49 & $309,6065^{* *}$ & $21,3946^{* *}$ & $5,2014^{* *}$ & $7,4484^{* *}$ \\
Resíduos & 98 & 11,8677 & 6,1991 & 3,0027 & 0,4048 \\
CV $(\%)$ & - & 15,6685 & 7,3299 & 2,9692 & 20,0396 \\
\hline
\end{tabular}

FV - Fontes de Variação; Pr - Produtividade (t ha-1 ${ }^{1}$; MS - Matéria Seca (\%); TA - Teor de amido (\%); RE: Rendimento em etanol $\left(\mathrm{m}^{3}\right.$ ha- $\left.^{-1}\right)$; **significativo pelo teste de $\mathrm{F}(\mathrm{p}<0,01)$ 
Tabela 2 - Médias de quatro características avaliadas em 50 clones de batata doce pelo método de Scott e Knott. Palmas-TO, 2010

\begin{tabular}{|c|c|c|c|c|}
\hline \multirow{2}{*}{ Clones } & \multicolumn{4}{|c|}{ CARACTERÍSTICAS } \\
\hline & $\operatorname{Pr}$ & MS & $\mathrm{TA}$ & $\mathrm{RE}$ \\
\hline 02.21 & $19,114 \mathrm{e}$ & $34,509 \mathrm{a}$ & $58,043 \mathrm{a}$ & $2,757 \mathrm{~d}$ \\
\hline 02.26 & $22,437 \mathrm{~d}$ & $36,543 \mathrm{a}$ & 57,629 a & $3,338 \mathrm{~d}$ \\
\hline 02.37 & $20,291 \mathrm{e}$ & $33,193 \mathrm{~b}$ & $59,2 \mathrm{a}$ & $2,410 \mathrm{~d}$ \\
\hline 02.39 & $31,076 \mathrm{c}$ & $33,363 \mathrm{~b}$ & $54,59 \mathrm{a}$ & $4,250 \mathrm{c}$ \\
\hline 02.58 & $14,083 \mathrm{f}$ & $33,891 \mathrm{a}$ & $59,704 \mathrm{a}$ & $1,873 \mathrm{e}$ \\
\hline 04.06 & $11,191 \mathrm{f}$ & $33,131 \mathrm{~b}$ & $59,382 \mathrm{a}$ & $1,582 \mathrm{e}$ \\
\hline 04.12 & $12,38 \mathrm{f}$ & $35,214 \mathrm{a}$ & $59,232 \mathrm{a}$ & $1,856 \mathrm{e}$ \\
\hline 04.30 & $5,033 \mathrm{~g}$ & $32,613 \mathrm{~b}$ & $58,06 \mathrm{a}$ & $0,685 \mathrm{e}$ \\
\hline 100.06 & $9,319 \mathrm{f}$ & $34,346 \mathrm{a}$ & $58,031 \mathrm{a}$ & $1,376 \mathrm{e}$ \\
\hline 100.18 & $21,625 \mathrm{e}$ & $34,725 \mathrm{a}$ & $58,84 \mathrm{a}$ & $3,037 \mathrm{~d}$ \\
\hline 100.23 & $25,375 \mathrm{c}$ & $31,980 \mathrm{~b}$ & 58,614 a & $3,801 \mathrm{c}$ \\
\hline 100.32 & $24,305 \mathrm{~d}$ & $35,183 \mathrm{a}$ & $56,774 \mathrm{a}$ & $3,778 \mathrm{c}$ \\
\hline 106.10 & $31,75 \mathrm{~d}$ & $31,752 \mathrm{~b}$ & $57,825 \mathrm{a}$ & $3,277 \mathrm{~d}$ \\
\hline 106.25 & $12,245 \mathrm{f}$ & $24,531 \mathrm{~d}$ & 56,353 a & $1,310 \mathrm{e}$ \\
\hline 106.27 & $18,291 \mathrm{e}$ & $30,565 \mathrm{~b}$ & $59,174 \mathrm{a}$ & $2,246 \mathrm{~d}$ \\
\hline 106.32 & $14,154 \mathrm{f}$ & $34,234 \mathrm{a}$ & $58,974 \mathrm{a}$ & $2,055 \mathrm{e}$ \\
\hline 106.41 & $35,766 \mathrm{~b}$ & $35,535 \mathrm{a}$ & 59,498 a & $5,446 b$ \\
\hline 106.55 & $8,708 \mathrm{f}$ & $31,257 \mathrm{~b}$ & $56,981 \mathrm{a}$ & $1,315 \mathrm{e}$ \\
\hline 106.63 & $25,851 \mathrm{~d}$ & $37,742 \mathrm{a}$ & $60,486 \mathrm{a}$ & $4,240 \mathrm{c}$ \\
\hline 106.79 & $31,156 \mathrm{c}$ & $36,942 \mathrm{a}$ & 58,28 a & $4,826 \mathrm{c}$ \\
\hline 112.13 & $6,111 \mathrm{~g}$ & $33,441 \mathrm{~b}$ & 59,907 a & $1,087 \mathrm{e}$ \\
\hline 114.03 & $24,715 \mathrm{~d}$ & $34,945 \mathrm{a}$ & 56,198 a & $3,514 \mathrm{c}$ \\
\hline 114.07 & $17,318 \mathrm{e}$ & $35,768 \mathrm{a}$ & 59,806 a & $2,705 \mathrm{~d}$ \\
\hline 114.08 & $5,370 \mathrm{~g}$ & $32,434 \mathrm{~b}$ & $57,424 \mathrm{a}$ & $1,197 \mathrm{e}$ \\
\hline 114.15 & $20,145 \mathrm{e}$ & $28,400 \mathrm{c}$ & 58,82 a & $2,447 d$ \\
\hline 114.22 & $24,840 \mathrm{~d}$ & $32,807 \mathrm{~b}$ & $60,47 \mathrm{a}$ & $3,721 \mathrm{c}$ \\
\hline 115.01 & $23,804 \mathrm{~d}$ & $36,730 \mathrm{a}$ & $57,8267 \mathrm{a}$ & $3,640 \mathrm{c}$ \\
\hline 115.02 & $4,416 \mathrm{~g}$ & $34,781 \mathrm{a}$ & $58,573 \mathrm{a}$ & $0,648 \mathrm{e}$ \\
\hline 22.06 & $9,218 \mathrm{f}$ & $35,489 \mathrm{a}$ & $60,623 \mathrm{a}$ & $1,150 \mathrm{e}$ \\
\hline 22.14 & $21, \mathrm{e}$ & 34,449 a & $56,827 \mathrm{a}$ & $2,978 \mathrm{~d}$ \\
\hline 22.19 & $45,473 \mathrm{a}$ & 38,359 a & 59,69 a & $7,638 \mathrm{a}$ \\
\hline 22.24 & 18,659 e & $36,080 \mathrm{a}$ & 58,255 a & $2,544 \mathrm{~d}$ \\
\hline 22.32 & $24,145 \mathrm{~d}$ & $35,293 \mathrm{a}$ & $57,683 \mathrm{a}$ & $2,838 \mathrm{~d}$ \\
\hline 48.14 & $26,625 \mathrm{~d}$ & $28,868 \mathrm{c}$ & $56,797 \mathrm{a}$ & $3,269 \mathrm{~d}$ \\
\hline Amanda & $38,402 \mathrm{~b}$ & $31,404 \mathrm{~b}$ & 57,868 a & $5,033 \mathrm{~b}$ \\
\hline A.Clara & $31,942 \mathrm{c}$ & $32,212 \mathrm{~b}$ & 58,226 a & $4,302 \mathrm{c}$ \\
\hline Barbara & $39,087 \mathrm{~b}$ & 35,736 a & 59,733 a & $6,050 \mathrm{~b}$ \\
\hline Beatriz & $30,104 \mathrm{c}$ & $32,751 \mathrm{~b}$ & 58,736 a & $4,153 \mathrm{c}$ \\
\hline C.Vitoria & $33,519 b$ & $37,534 \mathrm{a}$ & $58,487 \mathrm{a}$ & $5,33 \mathrm{~b}$ \\
\hline Duda & $40,912 \mathrm{a}$ & $36,617 \mathrm{a}$ & $55,363 \mathrm{a}$ & $5,975 \mathrm{~b}$ \\
\hline
\end{tabular}


Continuação da Tabela 2

\begin{tabular}{lllll}
\hline Isabela & $21,641 \mathrm{e}$ & $31,666 \mathrm{~b}$ & $58,41 \mathrm{a}$ & $2,867 \mathrm{~d}$ \\
Julia & $23,611 \mathrm{~d}$ & $33,211 \mathrm{~b}$ & $57,75 \mathrm{a}$ & $3,226 \mathrm{~d}$ \\
Livia & $32,361 \mathrm{c}$ & $36,629 \mathrm{a}$ & $60,416 \mathrm{a}$ & $5,124 \mathrm{~b}$ \\
Marcela & $35,895 \mathrm{~b}$ & $35,970 \mathrm{a}$ & $59,878 \mathrm{a}$ & $5,574 \mathrm{~b}$ \\
PA.06 & $19,562 \mathrm{e}$ & $31,914 \mathrm{~b}$ & $57,01 \mathrm{a}$ & $1,966 \mathrm{e}$ \\
PA.18 & $26,123 \mathrm{~d}$ & $39,133 \mathrm{a}$ & $59,563 \mathrm{a}$ & $4,384 \mathrm{c}$ \\
PA.32 & $10,5 \mathrm{f}$ & $34,205 \mathrm{a}$ & $57,731 \mathrm{a}$ & $2,598 \mathrm{~d}$ \\
PA.35 & $13,604 \mathrm{f}$ & $33,744 \mathrm{a}$ & $58,385 \mathrm{a}$ & $2,024 \mathrm{e}$ \\
PA.36 & $10,718 \mathrm{f}$ & $31,230 \mathrm{~b}$ & $58,29 \mathrm{a}$ & $1,302 \mathrm{e}$ \\
PA.57 & $28,333 \mathrm{c}$ & $35,321 \mathrm{a}$ & $57,548 \mathrm{a}$ & $3,992 \mathrm{c}$ \\
\hline
\end{tabular}

Pr - Produtividade (t ha-1 ${ }^{1}$; MS - Matéria Seca (\%); TA - Teor de amido (\%); RE: Rendimento em etanol ( $\mathrm{m}^{3}$ ha- $\left.^{-1}\right)$; As médias seguidas de pela mesma letra, na coluna, não diferem entre si, pelo teste Scott e Knott $(\mathrm{p}<0,05)$

Nos teores médios de amido (Tabela 2), os clones 22.06, 106.63, 114.22 e Lívia apresentaram os maiores percentuais, acima de $60 \%$. Os clones Duda e 02.39 , com teores médios abaixo de 55,3\%, apresentaram os menores valores de amido. Oliveira et al. (2005) trabalhando com diferentes níveis de uréia na adubação de batata doce encontraram como valor mínimo, 57\% de amido. Pavlak et al. (2007) utilizando a farinha do mesocarpo do babaçu para a produção de etanol obtiveram aproximadamente $52 \%$ de amido. Bringhenti e Cabello (2005) trabalhando com a produção de álcool a partir da mandioca, encontraram $81,31 \%$ de amido.

No rendimento em etanol foi observada a formação de cinco grupos (Tabela 2). No primeiro grupo, tem destaque o clone 22.19 , com o maior rendimento em etanol $\left(7,63 \mathrm{~m}^{3} \mathrm{ha}^{-1}\right)$. No segundo grupo, o clone Barbara apresentou o maior rendimento $\left(6,05 \mathrm{~m}^{3} \mathrm{ha}^{-1}\right)$. O terceiro grupo, formado por 12 clones, apresentou rendimentos oscilando de 3,51 a 4,82 $\mathrm{m}^{3}$ ha $^{-1}$. O quarto grupo com 15 clones e o quinto grupo, também com 15 clones, foram os que obtiveram as menores médias para esta característica.

No presente trabalho, a análise de agrupamento das médias colocou no mesmo grupo, os três clones com maior percentagem média de amido e matéria seca. Segundo Leonel e Cereda (2002) a matéria seca é de grande importância para a indústria, pois o alto teor desta é vantajoso por proporcionar um maior rendimento de extração de amido. O clone 22.19, apesar de não apresentar os maiores percentuais médios, se destacou por estar presente nos grupos com os maiores valores de produtividade, matéria seca, teor de amido e rendimento em etanol.

O método de agrupamento proposto por Tocher, a partir das distâncias generalizadas de Mahalanobis (1936), possibilitou a divisão dos 50 materiais em oito grupos geneticamente disjuntos (Tabela 3).

Tabela 3 - Agrupamento estabelecido pelo método de Tocher entre 50 clones de batata doce avaliados quanto a quatro características, tendo como medida de dissimilaridade a distância generalizada de Mahalanobis. Palmas-TO, 2010

\begin{tabular}{cc}
\hline Grupos & Clones \\
\hline I & 106.41; Marcela; Barbara; C. Vitoria; Livia; 106.79; Beatriz; A. Clara; PA. 57 100.23; 106.63; PA.18; 115.01; \\
II & $100.32 ; 114.03 ; 114.22 ;$ Julia; 02.26; 106.10; 100.18 \\
III & $02.21 ; 22.24 ; 22.14 ;$ Isabela; $02.37 ; 106.27 ;$ PA.06; 22.32; $114.15 ; 48.14$ \\
IV & $02.39 ;$ Amanda \\
V & Duda \\
VI & 22.19 \\
VII & 106.25 \\
VIII & PA.32 \\
\hline
\end{tabular}


No grupo 1 foram incluídos 20 clones geneticamente similares ( $40 \%$ do total), o que indica que possíveis cruzamentos entre esses clones reduzirá, teoricamente, a obtenção de materiais superiores. O segundo grupo foi formado por 14 clones ( $28 \%$ do total), o terceiro por 10 clones, o quarto por 2 clones e os demais grupos, formados por um clone cada.

A formação desses grupos representa valiosa informação na escolha de genitores dentro dos programas de melhoramento, pois as novas populações híbridas a serem estabelecidas devem ser embasadas na magnitude de suas dissimilaridades e no potencial "per se" dos genitores. Com o emprego desses clones em futuros cruzamentos, há grande possibilidade de obter populações segregantes superiores, sendo uma informação muito útil aos programas de melhoramento (BERTAN et al., 2006).

Os clones reunidos em grupos mais distantes dão um indicativo de serem dissimilares, podendo ser consideradas como promissores em cruzamentos artificiais. Contudo, apesar da divergência, tais clones devem associar média elevada e variabilidade para os caracteres avaliados.

A análise de comparação de médias, juntamente com o agrupamento estabelecido pelo método de Tocher, permite a identificação de quais serão os cruzamentos promissores, e os que poderão resultar em variabilidade restrita nas gerações segregantes, como aqueles realizados entre progenitores de um mesmo grupo. Neste sentido, poderão ser esperadas como promissoras as seguintes hibridações: Duda x 22.19, Marcela x 22.19, Barbara x 22.19, Duda x Barbara, Amanda x 22.19, Amanda x Marcela, pois foram dissimilares (Tabela 3) e apresentaram médias per se elevadas para as características avaliadas (Tabela 2). Assim, quando utilizadas em hibridações dirigidas em programa de melhoramento genético, possibilitarão ampliar o número de recombinantes desejáveis, a fim de que possam ser utilizadas como fontes de obtenção de constituições genéticas superiores.

$\mathrm{Na}$ Tabela 4, a contribuição relativa de cada característica para a dissimilaridade genética, segundo método de Singh (1981), aponta que duas características contribuíram com mais de $85 \%$ da divergência genética e as outras duas contribuíram com pouco mais de $14 \%$, sendo que destas, o teor de amido contribuiu com apenas 5,14\%.

A matéria seca também contribuiu pouco para a divergência, apenas $9,64 \%$, embora tenha apresentado grande variabilidade. Já os caracteres produtividade $(70,15 \%)$ e o rendimento em etanol $(15,06 \%)$ foram as mais eficientes em explicar a dissimilaridade entre os clones, devendo com isso ser priorizadas na escolha de genitores em programas de melhoramento.
Tabela 4 - Contribuição relativa de quatro características para a dissimilaridade genética de 50 clones de batata doce. Método proposto por Singh (1981). Palmas-TO, 2010

\begin{tabular}{lc}
\hline \multicolumn{1}{c}{ Características } & Contribuição Relativa (\%) \\
\hline Produtividade & 70,1542 \\
Rendimento em etanol & 15,0609 \\
Matéria Seca & 9,6371 \\
Teor de Amido & 5,1478 \\
\hline
\end{tabular}

\section{CONCLUSÕES}

1. Os clones apresentaram-se divergentes quanto à produtividade, rendimento em etanol, matéria seca e teor de amido;

2. As características produtividade $(70,15 \%)$ e rendimento em etanol $(15,06 \%)$ foram as que mais contribuíram na divergência genética entre os clones;

3. Os cruzamentos Duda x 22.19, Marcela x 22.19, Barbara x 22.19, Duda x Barbara, Amanda x 22.19, Amanda x Marcela são promissores para obtenção de híbridos divergentes, já que estes se apresentaram de forma dissimilar em todas as características.

\section{AGRADECIMENTOS}

Ao $\mathrm{CNPq}$ pela concessão da bolsa; à Secretaria de Ciência e Tecnologia do Estado do Tocantins pelo fomento; à Universidade Federal do Tocantins; à equipe do Laboratório de Biotecnologia da UFT e LASPER pelo auxilio na realização do trabalho.

\section{REFERENCIAS}

ANDRADE JUNIOR, V. C. et al. Selection of sweet potato clones for the region Alto Vale do Jequitinhonha. Horticultura Brasileira, v. 27, n. 03, p. 389-393, 2009.

ASSOCIATION OF OFFICIAL ANALYTICAL CHEMISTS. Methods of analysis of the Association of official Analytical Chemists. 12 ed. Washington, 1994. 1094 p.

BERTAN, I. et al. Dissimilaridade genética entre genótipos de trigo avaliados em cultivo hidropônico sob estresse por alumínio. Bragantia, v. 65, n. 01, p. 55-63, 2006.

BRINGHENTI, L.; CABELLO, C. Qualidade do álcool produzido a partir de resíduos amiláceos da agroindustrialização da mandioca. Revista Energia na Agricultura, v. 20, n. 04, p. 36-52, 2005. 
CARDOSO, A. D. et al. . Avaliação de clones de batata doce em Vitória da Conquista. Horticultura Brasileira, v. 23, n. 04, p. 911-914, 2005.

CRUZ C. D. Programa Genes: Aplicativo computacional em genética e estatística. Viçosa: UFV, 2007.

LEAL, M. R. L. V. et al. Outras matérias-primas para a produção de etanol. In: CORTEZ, Luís Augusto Barbosa (Org.). Bioetanol de Cana-de-Açúcar: $P \& D$ para produtividade e Sustentabilidade. São Paulo: Blucher, 2010. p. 519-539. v. 01.

LEONEL, M.; CEREDA, M. P. Caracterização físico-química de algumas tuberosas amiláceas. Ciência e Tecnologia de Alimentos, v. 22, n. 01, p. 65-69, 2002.

MAHALANOBIS, P. C. On the generalized distance in statistics. National Academy of Sciences, India Proceedings, v. 02, p. $49-55,1936$.

OLIVEIRA, A. P. et al. Rendimento e qualidade de raízes de batata-doce adubada com níveis de uréia. Horticultura Brasileira, v. 23, n. 04, p. 925-928, 2005.

PAVLAK, M. C. M. et al. Aproveitamento da farinha do mesocarpo do babaçu (Orbignya martiana) para obtenção de etanol. Evidência, v. 07, n. 01, p. 07-24, 2007.
RAO, C. R. Advanced statistical methods in biometric research. New York: John Willey, 1952. 390 p.

ROESLER, P. V. S. de O. et al. Produção e qualidade de raiz tuberosa de cultivares de batata-doce no oeste do Paraná. Acta Scientiarum Agronomy, v. 30, n. 01, p. 117-122, 2008.

SALLA, D. A.; CABELLO, C. Análise energética de sistemas de produção de etanol de mandioca, cana-de-açúcar e milho. Revista Energia na Agricultura, v. 25, n. 02, p. 32-53, 2010,

SANTOS, J. F. et al. Produção de batata-doce adubada com esterco bovino em solo com baixo teor de matéria orgânica. Horticultura Brasileira, v. 24, p. 103-106, 2006.

SINGH, D. The relative importance of characters affecting genetic divergence. The Indian Journal of Genetic and a Plant Breeding, v. 41, n. 02, p. 237-245, 1981

SCOTT, A. J.; KNOTT, M. A. A cluster analysis method for grouping means in the analysis of variance. Biometrics, v. 30, n. 03 , p. $507-512,1974$

SUAREZ, P. A. Z. et al. Transformação de triglicerídeos em combustíveis, materiais poliméricos e insumos químicos: algumas aplicações da catálise na oleoquímica. Química Nova, v. 30, n. 03, p. 667-676, 2007. 